\title{
GW23-e2574 OLMESARTAN AMELIORATES LEFT VENTRICULAR DIASTOLIC DYSFUNCTION IN SPONTANEOUSLY HYPERTENSIVE RATS THROUGH INHIBITING CALCINEURIN
}

doi:10.1136/heartjnl-2012-302920a.100

${ }^{1}$ Fu mingqiang, 'Zhou jingmin, ${ }^{1}$ Qian juying, ${ }^{2}$ Zhong chunlin, ${ }^{2}$ Jiang kun, 'Zou yunzen, ${ }^{1} \mathrm{Ge}$ junbo, ${ }^{1} \mathrm{Ge}$ junbo. ${ }^{1}$ Shanghai Institute of Cardiovascular Diseases, Department of Cardiology, Zhongshan Hospital, Fudan University; ${ }^{2}$ Department of Cardiology, the Affiliated Hospital of North Sichuan Medical College

Objectives To test whether treatment of olmesartan ameliorates cardiac diastolic dysfunction in spontaneously hypertensive rats (SHR) and whether this is calcineurin $(\mathrm{CaN})$ involved.

Methods Two groups of 6-month-old male SHR were treated with either saline $(n=6)$ or olmesartan $\left(2.5 \mathrm{mg} \mathrm{kg}^{-1}\right.$ day $\left.^{-1}, \mathrm{n}=6\right)$ for 3 months. Age matched Wistar-Kyoto rats (WKY, $n=6$ ) were also served as controls. Heart rate (HR), systolic blood pressure (SBP), cardiac structure and function, histological examinations and expression of $\mathrm{CaN}$ were all determined.

Results SHR of 6 months old exhibited evident cardiac hypertrophy and diastolic dysfunction as demonstrated by elevated systolic blood pressure, increased left ventricular mass index and decreased $\mathrm{E} / \mathrm{A}$ and $\mathrm{E}^{\prime} / \mathrm{A}^{\prime}$ while systolic function assessed by ejection fraction (EF) and fractional shortening (FS) remained unimpaired when compared with WKY controls. Treatment with olmesartan significantly decreased systolic blood pressure and ventricular hypertrophy, attenuated fibrosis and improved diastolic function in olmesartan group compared to saline $(p<0.05)$. Meanwhile, CaN expression was also downregulated after treatment in olmesartan group as compared with the other two groups (both $\mathrm{p}<0.05$ ).

Conclusions Our data suggest that the beneficial effect of olmesartan on cardiac structure and diastolic function may be, to some extent, through $\mathrm{CaN}$ pathway. 